\title{
Weighted weak type inequalities with variable exponents for Hardy and maximal operators
}

\author{
M. Isabel Aguilar Cañestro and Pedro Ortega Salvador \\ Análisis Matemático, Facultad de Ciencias, Universidad de Málaga, 29071 Málaga, Spain
}

(Communicated by Shigefumi MorI, M.J.A., Oct. 12, 2006)

\begin{abstract}
We characterize the weighted weak type inequalities with variable exponents for the modified Hardy operators and the Hardy-Littlewood maximal operators.

Key words: Hardy operators; Hardy-Littlewood maximal operator; variable $L^{p}$ spaces; weak type inequalities; weights.
\end{abstract}

1. Introduction. Let $(X, \mathcal{M}, \mu)$ and $(Y, \mathcal{N}, \nu)$ be measure spaces and let $T$ be a sublinear operator that transforms $\mathcal{M}$-measurable functions in $\mathcal{N}$ measurable functions. We say that $T$ is of weak type $(p, p), p \geq 1$, if there exists $K>0$ such that the inequality

$$
\nu\{y \in Y:|T f(y)|>\lambda\} \leq \frac{K}{\lambda^{p}} \int_{X}|f|^{p} d \mu
$$

holds for all $f$ and all $\lambda>0$.

The weak type inequalities are of interest in interpolation theory and play a fundamental role in the study of the almost everywhere convergence of sequences of sublinear operators [6].

In 1972, B. Muckenhoupt [12] proved an important result about the weak type inequalities for the Hardy-Littlewood maximal operator in $\boldsymbol{R}$, which is defined by

$$
M f(x)=\sup _{s, h>0} \frac{1}{s+h} \int_{x-s}^{x+h}|f(t)| d t .
$$

Muckenhoupt showed that if $u$ and $v$ are nonnegative measurable functions (weights), then the weighted weak type inequality

$$
\int_{\{x \in \boldsymbol{R}: M f(x)>\lambda\}} u \leq \frac{K}{\lambda^{p}} \int_{\boldsymbol{R}}|f(x)|^{p} v(x) d x
$$

holds for all $f$ and all $\lambda>0$ with a constant $K>0$ independent of $f$ and $\lambda$ if and only if $(u, v)$ verifies the condition $A_{p}$, which means that

$$
\sup _{a<b} \frac{1}{b-a}\left(\int_{a}^{b} u\right)\left(\operatorname{ess~sup}_{x \in(a, b)} v^{-1}(x)\right)<\infty
$$

2000 Mathematics Subject Classiffcation. 42B25, 26D10. if $p=1$ and

$$
\sup _{a<b} \frac{1}{b-a}\left(\int_{a}^{b} u\right)^{\frac{1}{p}}\left(\int_{a}^{b} v^{1-p^{\prime}}\right)^{\frac{1}{p^{\prime}}}<\infty
$$

if $p>1$, where $p^{\prime}=\frac{p}{p-1}$.

The theory of weighted weak type inequalities was subsequently extended by considering inequalities of the form

(1) $\int_{\{x \in \boldsymbol{R}: T f(x)>\lambda\}} u \leq \frac{K}{\Phi(\lambda)} \int_{\boldsymbol{R}} \Phi(|f(x)|) v(x) d x$,

where $\Phi$ is a Young function (see [7]). The characterizations of the pairs of weights $(u, v)$ such that the inequality (1) holds for the Hardy-Littlewood maximal operator and other operators as singular or fractional integrals can be found in [7] and in the references therein.

In last years, stimulated by some problems in fluid dynamics and in differential equations, a great interest has arised in the spaces $L^{p}$ with variable exponents (see [8] for the definitions) and in the extension of the results of classical Harmonic Analysis to the variable exponent setting. In particular, the boundedness of the Hardy-Littlewood maximal operator in spaces $L^{p}$ with variable exponents has been studied by several authors $([2,3,13])$.

In this setting, it is natural to consider the weighted weak type inequalities with variable exponents, as a new extension of the theory of classical weighted weak type inequalities.

By a weighted weak type inequality with variable exponent for an operator $T$ in a subset $A$ of $\boldsymbol{R}$ we understand an inequality of the form

$$
\int_{\{x \in A: T f(x)>\lambda\}} u \leq \int_{A}\left(\frac{K|f(x)|}{\lambda}\right)^{p(x)} v(x) d x
$$


where $p(\cdot)$ is a measurable function such that $p(x) \geq$ 1 for all $x \in A$. These inequalities are generalizations of the classical weighted weak type inequalities.

D. Cruz-Uribe, A. Fiorenza and C. J. Neugebauer [2] seem to be the first authors that have studied a weak type inequality with variable exponent, although with a different position of the constant with respect to the inequality (2) and without weights. They have shown that the Hardy-Littlewood maximal operator verifies the inequality

$$
|\{x \in \boldsymbol{R}: M f(x)>\lambda\}| \leq K \int_{\boldsymbol{R}}\left(\frac{|f(x)|}{\lambda}\right)^{p(x)} d x
$$

if there exists $K>0$ such that for all bounded intervals $I, \frac{1}{p(x)} \leq \frac{K}{|I|} \int_{I} \frac{1}{p(y)} d y$ a.e. $x \in I$.

We will characterize the pairs of weights $(u, v)$ such that the Hardy-Littlewood maximal operator verifies the inequality (2), obtaining a generalization of the classical Muckhenhoupt's Theorem [12]. We will also see that the unweighted weak type inequality with exponent $p(\cdot)$ for the Hardy-Littlewood maximal operator holds without any restrictions on the function $p$.

As previous steps, with independent interest, we will characterize the weighted weak type inequalities with variable exponents for the modified Hardy operators and the one-sided Hardy-Littlewood maximal operators. The work about the Hardy type operator will be done in section 2, while section 3 will be devoted to the maximal operators.

Throughout the paper, the letter $K$ will design a positive constant, not necessarily the same at each occurrence.

2. The modified Hardy operator. Let $-\infty \leq a<b \leq \infty$ and let $g$ be a positive nonincreasing function on $(a, b)$. The modified Hardy operator $T$ is defined for nonnegative functions $f$ on $(a, b)$ by $T f(x)=g(x) \int_{a}^{x} f$.

The weighted weak type inequalities for $T$ were characterized in $[1,5,11]$. Several papers have been written about the boundedness of Hardy type operators in variable $L^{p}$ spaces with weights (see, for instance, [4]). However, as far as we know, there are no papers in the literature about weighted weak type inequalities with variable exponents for Hardy type operators. In this section, we will characterize the pairs of weights $(u, v)$ such that the inequality

(3) $\int_{\{x \in(a, b): T f(x)>\lambda\}} u \leq \int_{a}^{b}\left(\frac{K f(x)}{\lambda}\right)^{p(x)} v(x) d x$ holds for all $f \geq 0$ and all $\lambda>0$ with a constant $K>0$ independent of $f$ and $\lambda$.

In the proof of our Theorem, the Young's inequality will play a fundamental role. It establishes that if $r>1$ and $r^{\prime}=\frac{r}{r-1}$, then $s t \leq \frac{s^{r}}{r}+\frac{t^{r^{\prime}}}{r^{\prime}}$ for all $s, t \geq 0$.

The result is the following one.

Theorem 1. Let $p:(a, b) \rightarrow[1, \infty)$. Let $P_{1}=\{x \in(a, b): p(x)=1\}$ and $P_{2}=\{x \in(a, b)$ : $p(x)>1\}$. Let $u, v$ be positive measurable functions on $(a, b)$. The following statements are equivalent:

(i) There exists $K>0$ such that (3) holds for all $f \geq 0$ and all $\lambda>0$.

(ii) There exists $K>0$ such that if $a<t<\beta<b$, then

$$
g\left(\beta^{-}\right)\left(\operatorname{essup}_{x \in P_{1} \cap(a, t)} v^{-1}(x)\right) \int_{t}^{\beta} u \leq K
$$

and

(5)

$$
\int_{P_{2} \cap(a, t)}\left(\frac{g\left(\beta^{-}\right)}{K}\right)^{p^{\prime}(x)}\left(\frac{\int_{t}^{\beta} u}{v(x)}\right)^{p^{\prime}(x)-1} d x \leq 1,
$$

where $p^{\prime}(y)=\frac{p(y)}{p(y)-1}$.

Moreover, if (i) holds with constant $K$, then (ii) holds with the same constant $K$ and if (ii) holds with constant $K$, then (i) holds with constant $8 K$.

Proof. (i) $\Rightarrow$ (ii) Let $a<t<\beta<b$. Let us prove (4). If $P_{1} \cap(a, t)$ has measure 0 or $g\left(\beta^{-}\right)=0$, there is nothing to prove. Suppose that $P_{1} \cap(a, t)$ has positive measure and $g\left(\beta^{-}\right)>0$. Let $\rho>0$ and let $F$ be a measurable subset of $P_{1} \cap(a, t)$ with positive measure such that $v(x) \leq \rho+\underset{y \in P_{1} \cap(a, t)}{\operatorname{essinf}} v(y)$ for all $x \in F$.

Let $f=\frac{1+\rho}{|F| g\left(\beta^{-}\right)} \chi_{F}$. If $x \in(t, \beta)$, then $T f(x) \geq$ $g(x) \frac{1+\rho}{|F| g\left(\beta^{-}\right)}|F| \geq 1+\rho>1$. The weak type inequality gives $g\left(\beta^{-}\right) \int_{t}^{\beta} u \leq K(1+\rho)\left(\rho+\operatorname{essinf}_{y \in P_{1} \cap(a, t)} v(y)\right)$, and letting $\rho$ tend to 0 , we get (4).

In order to prove (5), let $\left\{a_{n}\right\}$ be a strictly decreasing sequence with limit $a, \rho>0, n$ natural and $v_{n}=v+\frac{1}{n}$. Let, for each natural $k$, $P_{2}^{k}=P_{2} \cap\left\{x: p(x)>1+\frac{1}{k}\right\}$. Then, since $p^{\prime}$ is bounded above in $P_{2}^{k}$ and $v_{n}$ is bounded below, the integral 


$$
\varphi(\varepsilon)=\int_{P_{2}^{k} \cap\left(a_{n}, t\right)}\left(\frac{\varepsilon}{v_{n}(y)}\right)^{p^{\prime}(y)-1} g\left(\beta^{-}\right)^{p^{\prime}(y)} d y
$$

is finite for all $\varepsilon \geq 0$ and defines a continuous increasing function $\varphi$ such that $\varphi(0)=0$ and $\lim _{\varepsilon \rightarrow \infty} \varphi(\varepsilon)=\infty$. By continuity, there exists $\varepsilon_{0}>0$ such that $\varphi\left(\varepsilon_{0}\right)=(1+\rho) K$, where $K$ is the constant in the inequality (3).

Let $f(y)=\frac{1}{K}\left(\frac{\varepsilon_{0} g\left(\beta^{-}\right)}{v_{n}(y)}\right)^{p^{\prime}(y)-1} \chi_{P_{2}^{k} \cap\left(a_{n}, t\right)}(y)$. Let $x \in(t, \beta)$. Since $g$ is a nonincreasing function,

$$
\begin{aligned}
& T f(x)=g(x) \int_{P_{2}^{k} \cap\left(a_{n}, t\right)} \frac{1}{K}\left(\frac{\varepsilon_{0} g\left(\beta^{-}\right)}{v_{n}(y)}\right)^{p^{\prime}(y)-1} d y \\
& \geq \int_{P_{2}^{k} \cap\left(a_{n}, t\right)} \frac{1}{K}\left(\frac{\varepsilon_{0}}{v_{n}(y)}\right)^{p^{\prime}(y)-1} g\left(\beta^{-}\right)^{p^{\prime}(y)} d y \\
& =1+\rho>1 .
\end{aligned}
$$

Applying the weak type inequality to the function $f$ with $\lambda=1$ and taking into account that $v \leq v_{n}$, we obtain

$$
\begin{aligned}
& \int_{t}^{\beta} u \leq \int_{P_{2}^{k} \cap\left(a_{n}, t\right)}\left(\frac{\varepsilon_{0} g\left(\beta^{-}\right)}{v_{n}(y)}\right)^{\left(p^{\prime}(y)-1\right) p(y)} v(y) d y \\
& \leq \int_{P_{2}^{k} \cap\left(a_{n}, t\right)}\left(\frac{\varepsilon_{0} g\left(\beta^{-}\right)}{v_{n}(y)}\right)^{p^{\prime}(y)} v_{n}(y) d y \\
& =(1+\rho) K \varepsilon_{0} .
\end{aligned}
$$

Then,

$$
\begin{aligned}
& \int_{P_{2}^{k} \cap\left(a_{n}, t\right)}\left(\frac{g\left(\beta^{-}\right)}{K(1+\rho)}\right)^{p^{\prime}(y)}\left(\frac{\int_{t}^{\beta} u}{v_{n}(y)}\right)^{p^{\prime}(y)-1} d y \\
& \leq \int_{P_{2}^{k} \cap\left(a_{n}, t\right)}\left(\frac{g\left(\beta^{-}\right)}{K(1+\rho)}\right)^{p^{\prime}(y)}\left(\frac{K(1+\rho) \varepsilon_{0}}{v_{n}(y)}\right)^{p^{\prime}(y)-1} d y \\
& =1 .
\end{aligned}
$$

Letting $k$ tend to infinity, $\rho$ to 0 and $n$ to infinity, we obtain (5).

(ii) $\Rightarrow$ (i) We may assume that $f(x)>0$ for all $x \in(a, b)$ and $\int_{a}^{b} f<\infty$. Let $x_{0}=b$ and, given $x_{k}$, let $x_{k+1}$ be the unique point such that $\int_{a}^{x_{k+1}} f=\frac{1}{2} \int_{a}^{x_{k}} f$. In this way, we construct a sequence $\left\{x_{k}\right\}$ which decreases, has limit $a$ and verifies $\int_{a}^{x_{k}} f=4 \int_{x_{k+2}}^{x_{k+1}} f$ for all $k$. Let $O_{\lambda}=\{x \in(a, b)$ : $T f(x)>\lambda\}$ and $E_{k}=\left(x_{k+1}, x_{k}\right) \cap O_{\lambda}$.

If, for a fixed $k, E_{k}=\emptyset$, then its contribution to the integral $\int_{O_{\lambda}} u$ is null. Assume, therefore, that $k$ is a nonnegative integer such that $E_{k} \neq \emptyset$. If $x \in$
$E_{k}, \lambda<g(x) \int_{a}^{x} f \leq 4 g(x) \int_{x_{k+2}}^{x_{k+1}} f$. Since this holds for all $x \in E_{k}$, we have $\lambda \leq 4 g\left(\beta_{k}^{-}\right) \int_{x_{k+2}}^{x_{k+1}} f$, where $\beta_{k}=\sup E_{k}$. Then,

(6)

$$
\begin{aligned}
2 \int_{x_{k+1}}^{\beta_{k}} u & \leq \int_{x_{k+2}}^{x_{k+1}} 8 f(x) \frac{1}{\lambda} g\left(\beta_{k}^{-}\right)\left(\int_{x_{k+1}}^{\beta_{k}} u\right) d x \\
& =\int_{P_{1, k}}+\int_{P_{2, k}}=I+I I
\end{aligned}
$$

where $P_{1, k}=P_{1} \cap\left(x_{k+2}, x_{k+1}\right)$ and $P_{2, k}=P_{2} \cap$ $\left(x_{k+2}, x_{k+1}\right)$.

For the estimation of I, we apply (4) and obtain immediately

$$
\begin{aligned}
I & =\int_{P_{1, k}} \frac{8 K f(x)}{\lambda} \frac{v(x) g\left(\beta_{k}^{-}\right) v^{-1}(x)}{K}\left(\int_{x_{k+1}}^{\beta_{k}} u\right) d x \\
& \leq \int_{P_{1, k}}\left(\frac{8 K f(x)}{\lambda}\right) v(x) d x .
\end{aligned}
$$

On the other hand, Young's inequality and (5) yield

(8)

$$
\begin{aligned}
I I & =\int_{P_{2, k}} \frac{8 K f(x)}{\lambda} \frac{g\left(\beta_{k}^{-}\right) \int_{x_{k+1}}^{\beta_{k}} u}{K v(x) p(x)} v(x) p(x) d x \\
& \leq \int_{P_{2, k}}\left(\frac{8 K f(x)}{\lambda}\right)^{p(x)} v(x) d x \\
& +\int_{P_{2, k}}\left(\frac{g\left(\beta_{k}^{-}\right) \int_{x_{k+1}}^{\beta_{k}} u}{K v(x) p(x)}\right)^{p^{\prime}(x)} \frac{v(x) p(x)}{p^{\prime}(x)} d x \\
& \leq \int_{P_{2, k}}\left(\frac{8 K f(x)}{\lambda}\right)^{p(x)} v(x) d x \\
& +\int_{P_{2, k}}\left(\frac{g\left(\beta^{-}\right)}{K}\right)^{p^{\prime}(x)} \frac{\left(\int_{x_{k+1}}^{\beta_{k}} u\right)^{p^{\prime}(x)}}{v(x)^{p^{\prime}(x)-1} d x} \\
& \leq \int_{P_{2, k}}\left(\frac{8 K f(x)}{\lambda}\right)^{p(x)} v(x) d x+\int_{x_{k+1}}^{\beta_{k}} u .
\end{aligned}
$$

Transporting the estimations (7) and (8) to (6), we obtain

$$
\int_{x_{k+1}}^{\beta_{k}} u \leq \int_{x_{k+2}}^{x_{k+1}}\left(\frac{8 K f(x)}{\lambda}\right)^{p(x)} v(x) d x,
$$

and summing up in $k$, we get the inequality (3).

3. The Hardy-Littlewood maximal operators. Let us consider the one-sided HardyLittlewood maximal operator $M^{-}$defined by

$$
M^{-} f(x)=\sup _{h>0} \frac{1}{h} \int_{x-h}^{x}|f(t)| d t .
$$


The weighted weak type inequalities for $M^{-}$ were characterized by E. Sawyer [14], F. J. MartínReyes, P. Ortega and A. de la Torre [10] and F. J. Martín-Reyes [9]. They all showed that if $r \geq 1$, then the weighted weak type $(r, r)$ inequality for $M^{-}$with weights $u$ and $v$ holds if and only if $(u, v)$ verifies the condition $A_{r}^{-}$, which means that

$$
\sup _{a<b<c} \frac{1}{c-a}\left(\int_{b}^{c} u\right)^{\frac{1}{r}}\left(\int_{a}^{b} v^{1-r^{\prime}}\right)^{\frac{1}{r^{\prime}}}<\infty
$$

if $r>1$ and

$$
\sup _{a<b<c} \frac{1}{c-a}\left(\int_{b}^{c} u\right)\left(\underset{x \in(a, b)}{\operatorname{esssup} v^{-1}}(x)\right)<\infty
$$

if $r=1$.

We will apply Theorem 1 in order to characterize the pairs of weights $(u, v)$ such that the inequality

$$
\int_{\left\{x \in \boldsymbol{R}: M^{-} f(x)>\lambda\right\}} u \leq \int_{\boldsymbol{R}}\left(\frac{K|f(x)|}{\lambda}\right)^{p(x)} v(x) d x
$$

holds with a constant $K$ independent of $f$ and $\lambda$. The result reads as follows:

Theorem 2. Let $p: \mathbf{R} \rightarrow[1, \infty)$. Let $P_{1}=$ $\{x \in \mathbf{R}: p(x)=1\}$ and $P_{2}=\{x \in \mathbf{R}: p(x)>1\}$. Let $u, v$ be positive measurable functions on $\mathbf{R}$. The following statements are equivalent:

(i) There exists $K>0$ such that (9) holds for all $f$ and all $\lambda>0$.

(ii) The pair $(u, v)$ verifies the condition $A_{p(\cdot)}^{-}$, which means that there exists $K>0$ such that if $a<t<\beta$, then

$$
\frac{1}{\beta-a}\left(\operatorname{ess~sup}_{x \in P_{1} \cap(a, t)} v^{-1}(x)\right) \int_{t}^{\beta} u \leq K
$$

and

$$
\int_{(a, t) \cap P_{2}}\left(\frac{1}{K(\beta-a)}\right)^{p^{\prime}(x)}\left(\frac{\int_{t}^{\beta} u}{v(x)}\right)^{p^{\prime}(x)-1} d x \leq 1 .
$$

Moreover, if (i) holds with constant $K$, then (ii) holds with the same constant $K$ and if (ii) holds with constant $K$, then (i) holds with constant $8 K$.

Proof. (i) $\Rightarrow$ (ii) Let $a<t<\beta$ and let $f$ be a nonnegative function supported on $(a, \beta)$. If $x \in$ $(a, \beta)$, then $M^{-} f(x) \geq \frac{1}{x-a} \int_{a}^{x} f$. Since $M^{-}$verifies (i), we have

$$
\begin{aligned}
& \int_{\left\{x \in(a, \beta): \frac{1}{x-a} \int_{a}^{x} f>\lambda\right\}} u \leq \int_{\left\{x \in \boldsymbol{R}: M^{-} f(x)>\lambda\right\}} u \\
& \leq \int_{a}^{\beta}\left(\frac{K f(x)}{\lambda}\right)^{p(x)} v(x) d x .
\end{aligned}
$$

Hence, the modified Hardy operator $T_{a, \beta} f(x)=$ $\frac{1}{x-a} \int_{a}^{x} f, x \in(a, \beta)$, verifies (i) in Theorem 1 with a constant independent of $a$ and $\beta$. Therefore, by Theorem 1, (ii) holds.

(ii) $\Rightarrow$ (i) We may assume, without loss of generality, that $f$ has bounded below support. Then, the set $O_{\lambda}=\left\{x \in \boldsymbol{R}: M^{-} f(x)>\lambda\right\}$ is a bounded below open set. Let $\left\{\left(a_{j}, b_{j}\right)\right\}$ be the collection of the connected components of $O_{\lambda}$. It is well known that each $\left(a_{j}, b_{j}\right)$ verifies $\lambda<\frac{1}{x-a_{j}} \int_{a_{j}}^{x} f$ for all $x \in\left(a_{j}, b_{j}\right)$.

So, applying the condition (ii) and Theorem 1 to each operator $T_{j} f(x)=\frac{1}{x-a_{j}} \int_{a_{j}}^{x} f$, we obtain

$$
\begin{aligned}
\int_{O_{\lambda}} u & =\sum_{j=1}^{\infty} \int_{a_{j}}^{b_{j}} u=\sum_{j=1}^{\infty} \int_{\left\{x \in\left(a_{j}, b_{j}\right): \frac{1}{x-a_{j}} \int_{a_{j}}^{x} f>\lambda\right\}} u \\
& \leq \sum_{j=1}^{\infty} \int_{a_{j}}^{b_{j}}\left(\frac{8 K f(x)}{\lambda}\right)^{p(x)} v(x) d x \\
& \leq \int_{\boldsymbol{R}}\left(\frac{8 K f(x)}{\lambda}\right)^{p(x)} v(x) d x .
\end{aligned}
$$

It is worth noting that the condition $A_{p(\cdot)}^{-}$agrees with $A_{p}^{-}$when $p(x)$ is constant $(p(x) \equiv p)$.

A similar theorem can be proved for the operator $\mathrm{M}^{+}$defined obviously. In this case, the characterizing condition is $A_{p(\cdot)}^{+}$, whose formulation follows the same pattern as $A_{p(\cdot)}^{-}$, but with the opposite orientation.

As a consequence of Theorem 2 and the above observation, we will get the result for the HardyLittlewood maximal operator. It generalizes Muckenhoupt's Theorem [12] and reads as follows:

Theorem 3. Let $p, P_{1}, P_{2}, u$ and $v$ be as in Theorem 2. The following statements are equivalent:

(i) There exists $K>0$ such that the inequality

$$
\int_{\{x \in \mathbf{R}: M f(x)>\lambda\}} u \leq \int_{\mathbf{R}}\left(\frac{K|f(x)|}{\lambda}\right)^{p(x)} v(x) d x
$$

holds for all $f$ and all $\lambda>0$.

(ii) The pair $(u, v)$ verifies the condition $A_{p(\cdot)}$, which means that there exists $K>0$ such that if $a<b$, then 


$$
\frac{1}{b-a}\left(\operatorname{esssup}_{x \in P_{1} \cap(a, b)} v^{-1}(x)\right) \int_{a}^{b} u \leq K
$$

and

$$
\int_{P_{2} \cap(a, b)}\left(\frac{1}{K(b-a)}\right)^{p^{\prime}(x)}\left(\frac{\int_{a}^{b} u}{v(x)}\right)^{p^{\prime}(x)-1} d x \leq 1 .
$$

(iii) The pair $(u, v)$ verifies the conditions $A_{p(\cdot)}^{-}$and $A_{p(\cdot)}^{+}$.

Moreover, if (i) holds with constant $K$, then (ii) and (iii) hold with constant $K$ and if (ii) or (iii) holds with constant $K$, then (i) holds with constant $8 K$.

Proof. The proof of (i) $\Rightarrow$ (ii) is similar to the corresponding implication in Theorem 1 . The implication (ii) $\Rightarrow$ (iii) is trivial and (iii) $\Rightarrow$ (i) follows immediately from Theorem 2 and the fact that $\frac{1}{2}\left(M^{+} f+\right.$ $\left.M^{-} f\right) \leq M f \leq M^{+} f+M^{-} f$.

Finally, since the pair $(u, v)$ with $u=v=1$ verifies the condition $A_{p(\cdot)}$, the unweighted weak type inequality with exponent $p(\cdot)$ for the Hardy-Littlewood maximal operator holds.

Corollary 1. Let $p: \mathbf{R} \rightarrow[1, \infty)$. Then, there exists $K>0$ such that the inequality

$$
|\{x \in \mathbf{R}: M f(x)>\lambda\}| \leq \int_{\mathbf{R}}\left(\frac{K|f(x)|}{\lambda}\right)^{p(x)} d x
$$

holds for all $f$ and all $\lambda>0$.

This result can also be easily deduced from the classical weak type $(1,1)$ inequality for the HardyLittlewood maximal operator.

Acknowledgements. The authors have to thank the referee for some valuable comments and suggestions.

This research has been supported in part by MEC, grant MTM 2005-08350-C03-02, and Junta de Andalucía, grant FQM354.

\section{References}

[ 1 ] K. F. Andersen and B. Muckenhoupt, Weighted weak type Hardy inequalities with applications to Hilbert transforms and maximal functions, Studia Math. 72 (1982), 9-26.

[ 2 ] D. Cruz-Uribe, A. Fiorenza and C. J. Neugebauer, The maximal function on variable $L^{p}$ spaces, Ann. Acad. Sci. Fenn. Math. 28 (2003), 223238.

[ 3 ] L. Dienig, Maximal functions on generalized $L^{p(x)}$ spaces, Math. Inequal. Appl. 7 (2004), 245-253.

[ 4 ] D. E. Edmunds, V. Kokilashvili and A. Meskhi, On the boundedness and compactness of weighted Hardy operators in spaces $L^{p(x)}$, Georgian Math. J. 12(1) (2005), 27-44.

[ 5 ] E. V. Ferreyra, Weighted Lorentz norm inequalities for integral operators, Studia Math. 96 (1990), 125-134.

[ 6 ] A. Garsia, Topics in almost everywhere convergence, Markham Publishing Co., Chicago. Ill., 1970.

[ 7 ] V. Kokilashvili and M. Krbec, Weighted inequalities in Lorentz and Orlicz spaces, World Sci. Publishing, River Edge, NJ, 1991.

[ 8 ] O. Kovavcik and J. Rakosnik, On spaces $L^{p(x)}$ and $W^{k, p(x)}$, Czechoslovak Math. J. 41(116) (1991), no. 4, 592-618.

[ 9 ] F. J. Martín-Reyes, New proofs of weighted inequalities for one-sided Hardy-Littlewood maximal functions, Proc. Amer. Math. Soc. 117(3) (1993), 691-698.

[10 ] F. J. Martín-Reyes, P. Ortega Salvador and A. de la Torre, Weighted inequalities for one-sided maximal functions, Trans. Amer. Math. Soc. 319(2) (1990), 517-534.

[11 F. J. Martín-Reyes, P. Ortega and M. D. Sarrión, Boundedness of operators of Hardy type in $\Lambda^{p, q}$-spaces and weighted mixed inequalities for singular integral operators, Proc. Royal Soc. Edinburgh 127A (1997), 157-170.

[12 ] B. Muckenhoupt, Weighted norm inequalities for the Hardy maximal function, Trans. Amer. Math. Soc. 165 (1972), 207-226.

[13 A. Nekvinda, Hardy-Littlewood maximal operator on $L^{p(x)}\left(\mathbf{R}^{n}\right)$, Math. Inequal. Appl. 7 (2004), 255-265.

[14] E. T. Sawyer, Weighted inequalities for the onesided Hardy-Littlewood maximal functions, Trans. Amer. Math. Soc. 297 (1986), 53-61. 